\title{
Ultrafine measurements of the thermal shift of Fabry-Perot resonances
}

\author{
S. Michel*, F. Lemarquis and M. Lequime \\ Institut FRESNEL - UMR CNRS 6133 \\ Domaine Universitaire de Saint-Jérôme, 13397 Marseille Cedex 20 - France
}

\begin{abstract}
The wavelengths associated to the transmission peaks of a Fabry-Perot etalon are directly connected with its optical thickness. As a consequence, any change in the physical thickness or the refractive index of the cavity has a direct influence on the value of these specific wavelengths. This property can be used to determine the thermal characteristics, like the coefficient of thermal expansion or the thermo-optic coefficient, of some materials. However, to be efficient, this method needs a very high accuracy in the determination of these resonant wavelengths. We developed a dedicated measurement set-up, combining a tunable laser source around $850 \mathrm{~nm}$ and a high precision wavelength meter to determine with accuracy better than $0.2 \mathrm{pm}$ the resonant wavelengths of a Fabry-Perot etalon. We use a temperature stabilized chamber to avoid temperature fluctuations or, on the contrary, to apply a deterministic temperature change at the cavity level. We describe the structure of our bench and present the results obtained on BK7 windows as well as on an optically contacted ULE Fabry-Perot with silver coatings.
\end{abstract}

Keywords: Fabry-Perot interferometer, picometer resolution, coefficient of thermal expansion, thermo-optic coefficient

Corresponding authors: sebastien.michel@fresnel.fr

\section{INTRODUCTION}

Proposed for the first time by Charles Fabry (1867-1945) and Alfred Perot (1863-1925) in 1897, the Fabry-Perot is a multiple-beam interferometer, widely used in telecommunications, lasers, metrology and spectroscopy to control and measure the wavelength of light. The interference fringes given by this interferometer provides a high sensitivity to different effects applied to its structure. Indeed, it is sensitive, for instance, to a thermal change, or a mechanical stress applied on the interferometer's plates. This sensitivity can be used to determine thermal or mechanical properties of plates composing the interferometer [1,2]. The knowledge of these thermal properties for materials used in telecommunications or astronomy is currently a new challenge, to enhance the thermal stability of optical systems.

Therefore, we want to use the sensitivity of interferometry to determine with high accuracy the resonances of a FabryPerot, and then to access to the wavelength shifts with a temperature change. We aim to determine thermal properties of windows or to evaluate deformations on structures like substrates, which may be coated by a metallic thin film. We investigate a dedicated set-up in this aim, and we present our first results on BK7 window and an optically contacted Fabry-Perot interferometer.

\section{GENERALITIES AND FIRST APPROACH}

The Fabry-Perot interferometer is composed by two reflecting and parallel surfaces. Its transmittance function varies because of the interferences between multiple reflections of light that occurs between these two reflecting surfaces. Constructive interferences that correspond to high-transmission peaks of the etalon occur if the transmitted beams are in phase. We laid the bases of our study on these constructive interferences resulting from a Fabry-Perot interferometer composed by two non absorbing mirrors spaced by $60 \mu \mathrm{m}$ of air. For clarity, the notations we employed in this work, are summarized in Fig. 1, and Fig. 2 illustrates the associated transmittance spectrum.

In addition, in order to simplify the expressions, we consider two identical mirrors, so the transmittance and reflectance (in intensity) are the same for each mirror composing the interferometer. We note the transmittance (respectively reflectance) $T=T_{1}=T_{2}$ (respectively $R=R_{1}=R_{2}$ ). Accordingly, the phase change introduced by the reflections on each mirror is denoted by $\delta=\delta_{1}=\delta_{2}$.

The transmittance of the interferometer, described in Fig. 1, is therefore given by $[3,4]$ :

Optical Fabrication, Testing, and Metrology III, edited by Angela Duparré, Roland Geyl

Proc. of SPIE Vol. 7102, 71020Y · C 2008 SPIE · CCC code: 0277-786X/08/\$18 · doi: 10.1117/12.797710

Proc. of SPIE Vol. 7102 71020Y-1 


$$
T_{F P}=\frac{T_{M}}{1+m \sin ^{2} \phi},
$$

with $T_{\mathrm{M}}$ the maximum transmittance, $m$ the finesse of the cavity and $\phi$ the phase expressed as:

$$
\begin{aligned}
& T_{M}=\frac{T^{2}}{(1-R)^{2}}, \\
& m=\frac{4 R}{(1-R)^{2}}, \\
& \phi=\frac{2 \pi}{\lambda} n_{F P} D_{F P}-\delta .
\end{aligned}
$$

The resonances of the interferometer correspond to $\phi=0[\pi]$, and the wavelengths associated to the maximum of the transmittance function of the interferometer can be written like:

$$
\lambda_{p}=\frac{2 n_{F P} D_{F P}}{p+\frac{\delta}{\pi}}
$$

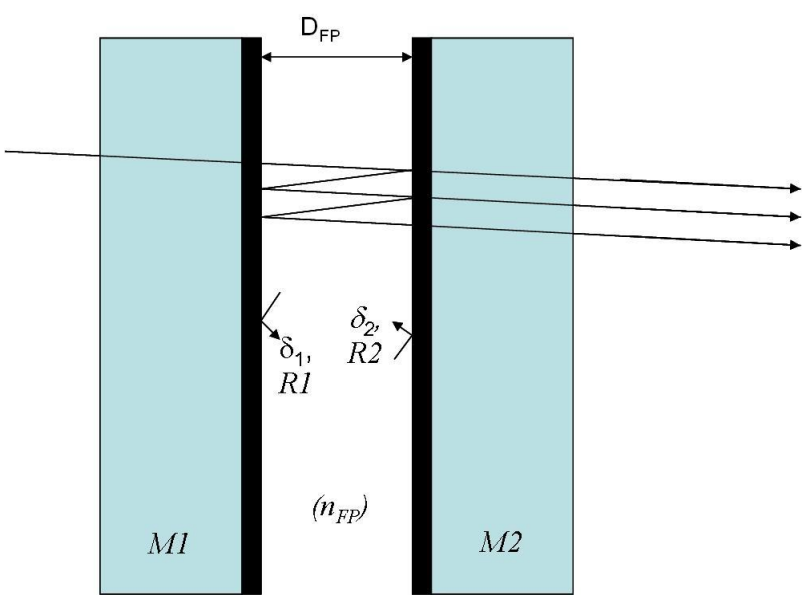

Figure 1: Notations used to describe the interferometer.

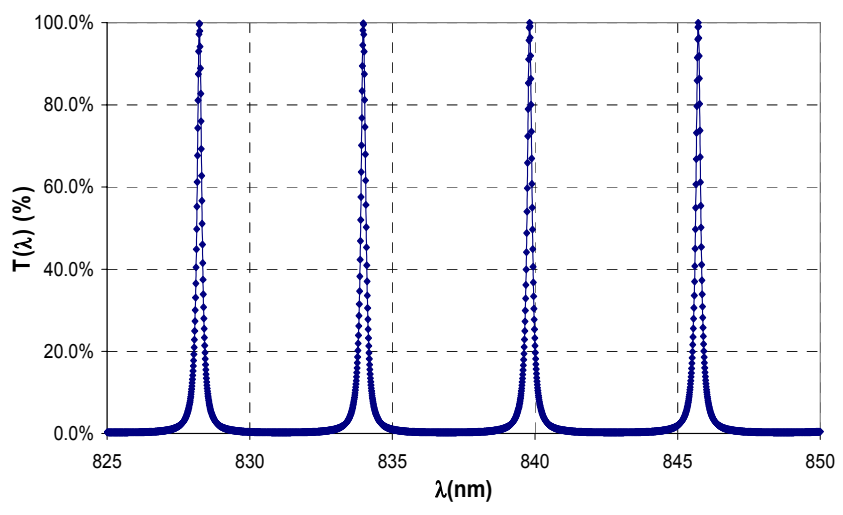

Figure 2: Transmittance spectrum of a non-absorbing Fabry-Perot interferometer. The spacer is a $60 \mu \mathrm{m}$ thick air layer. The value of transmittance and reflectance are $T=10 \%$ and $R=90 \%$ respectively.

Therefore, Eq.3 shows, first of all that the resonances are sensitive to the optical thickness of the cavity. Second of all, if the phase change $\delta$ and its variations, due to the reflection on the mirrors, are negligible in front of the others terms, Eq.3 can then be differentiated as follows:

$$
\frac{\Delta \lambda}{\lambda}=\frac{\Delta\left(D_{F P} n_{F P}\right)}{D_{F P} n_{F P}}=\frac{\Delta D_{F P}}{D_{F P}}+\frac{\Delta n_{F P}}{n_{F P}}
$$

The experimental set-up we want to develop is an optical bench with picometer accuracy on the determination of the global variations of optical thickness of a Fabry-Perot cavity. Considering a typical optical thickness of $60 \mu \mathrm{m}$ and an accuracy of $10 \mathrm{pm}$, this method shows that the expected accuracy on the wavelengths shift should be better than around $0.2 \mathrm{pm}$. Hence, we have to develop an experimental apparatus that would be capable in determining and measuring the resonances of a Fabry-Perot interferometer with an accuracy of at least $0.2 \mathrm{pm}$. 
In parallel, this common result has been confirmed by a theoretical study in which we simulate the behavior of a FabryPerot interferometer under a thermal change. Our numerical work takes into account every modification in thicknesses and refractive index of materials composing the interferometer, initiated by the thermal change $\Delta \theta$. It provides the resonances of the interferometer, and their associated shifts as a function of $\Delta \theta$. Besides, we decided to consider statistical noise to evaluate the consequences on the determination of the optical thickness of the cavity. Detailed results regarding this investigation are given in [5], however the main ones are recapitulated here:

- The access to an accuracy of about $10 \mathrm{pm}$ on the variation of the optical thickness of the cavity implies measurements of resonances and their shifts with a precision better than the picometer.

- If the relative variation of the cavity refractive index is known with an accuracy of $10^{-6}$, we can then evaluate the variation of the mechanical thickness with an accuracy of $10 \mathrm{pm}$. The Ciddor equation [6] allows this precise evaluation $\left(10^{-6}\right)$ of the refractive index variation of air.

- Simulations show that it is necessary to provide a measure of several channels, to enhance the tolerance on parallelism between mirrors: one at the centre of the interferometer, and the others regularly disposed around the first one. The utility of several channels is double. First, if the mirrors of interferometer have a defect of parallelism, then the average wavelengths shifts, measured simultaneously on each channel, provided the same results than if the mirrors had been perfectly parallel. Second, the regularly disposed channels around the centered one, are a mean to compare the effects of a thermal change between the centre and the other regions of the interferometer. Therefore, we can imagine that the interferometer presents, in its center, the coating (a circular disk shape) to be studied.

\section{POTENTIAL APPLICATIONS}

A high accurate measurement on the determination of optical thickness and its variations under a thermal change has numerous applications. We mainly apply our measurement to three different applications. The first one is the determination of the global temperature coefficient for a solid-spacer etalon. In this case, we have access to the $\alpha$ and

$\beta$ coefficient of the spacer, where $\alpha=\frac{1}{D} \frac{\partial D}{\partial \theta}$ and $\beta=\frac{1}{n} \frac{\partial n}{\partial \theta}$, with $D$ and $n$ respectively the thickness and the refractive index of the spacer.

The second one is the determination of the thermal expansion of a thin dielectric film deposited at the centre of the interferometer with the shape of a circular disk. The interferometer provides a reference channel (without the dielectric) and a measurement channel (with the dielectric). Nevertheless, the small thickness values (typically $1 \mu \mathrm{m}$ ) induces the effect of the thermal change $\Delta \theta$ to be weak on this latter (a few tens of picometer). Thus, the measurement is very sensitive to the thermal deformations, which cause a problem of reference. A precise study is needed to have the best accuracy on the thermal expansion of thin film, as described in [5].

The last application is to measure the optical thickness of an optically contacted Fabry-Perot and to evaluate its variations. In this case, the measurement provides a pure determination of the coefficient of thermal expansion (CTE) $\alpha$ of the material composing the spacer of the Fabry-Perot. Figures 3, 4 and 5 show the different structures on which we carried out measurements. As explained in Section 2, measurements on these structures need to be made at several points, we then choose to consider three channels, equally disposed around the center channel. 


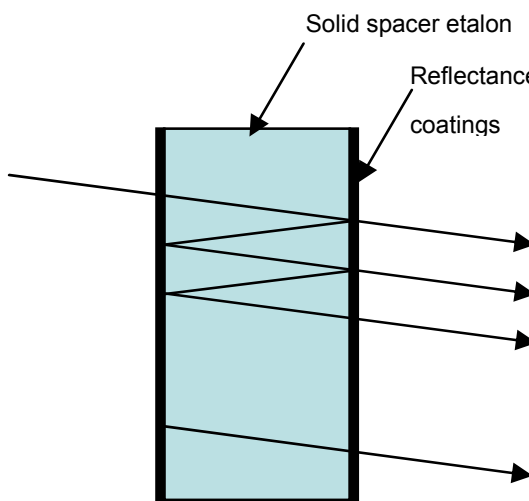

Figure 3: Determination of global variations of optical thickness on a solid spacer etalon under a thermal change

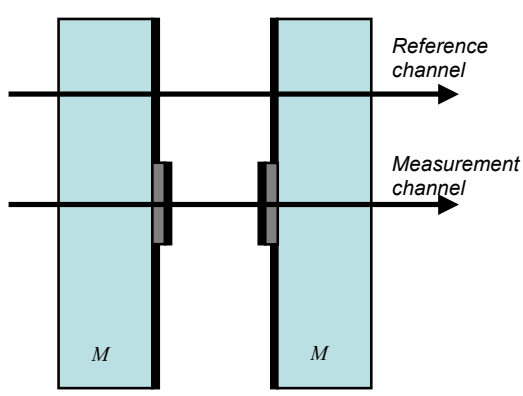

Figure 4: Determination of thermal expansion for a circular thin film disk deposited at the center of the interferometer

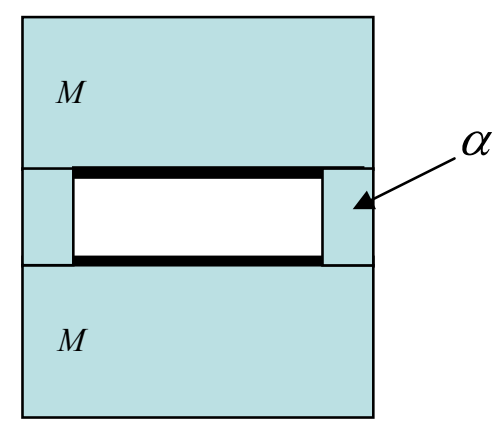

Figure 5: Determination of the CTE of material used for the spacer of the interferometer

\section{DEDICATED SET-UP FOR THE MEASUREMENT OF FABRY-PEROT RESONANCES}

We study several ways to evaluate the resonances of a Fabry-Perot, we describe here the one that provides the best results. Figure 6 shows the dedicated set-up we investigated to determine resonances of a Fabry-Perot interferometer with picometer accuracy. The basic principle is to estimate the transmittance $T$ versus the wavelength $\lambda$, with the help of a tunable laser. We thus access, point by point, to the transmittance spectrum of the interferometer. This measurement is done in the four channels included in the set-up. On each channel, we choose and fit a peak of the spectrum to precisely evaluate the average wavelength of resonance. Thereafter, we apply a deterministic thermal change $\Delta \theta$, and we follow the wavelengths shifts before evaluating the resulting $\Delta \lambda$.

We describe the various parts of our set-up in the following subsections:

\subsection{Emission system}

A tunable laser NEWFOCUS TLB6316 is used, in order to provide a very sharp laser line with an output power of around $10 \mathrm{~mW}$. This laser is tunable from $838 \mathrm{~nm}$ to $853 \mathrm{~nm}$, with a resolution of $20 \mathrm{pm}$ (coarse tuning mode). A piezoelectric transducer can be used to tune the laser in a restricted range of $150 \mathrm{pm}$ with a resolution less than $1 \mathrm{pm}$ (fine tuning mode). The laser beam is coupled into a single mode fiber (SMF) @ 850 nm. An optical coupler 99/1 divides the laser beam into two parts, $99 \%$ that goes directly for the sample illumination, and $1 \%$ are sent to a wavelength meter for a precise determination of the wavelength value.

\subsection{Illumination system}

The output of the SMF is positioned at the focal point of a best form lens to provide a collimated beam with a diameter of $20 \mathrm{~mm}$, which will illuminate the interferometer. This latter is thermally stabilized in a chamber manufactured by QUANTUM Northwest, which allows us to control the temperature stability down to $0.01^{\circ} \mathrm{C}$. This system permits also to apply a temperature change from $20^{\circ} \mathrm{C}$ to $80^{\circ} \mathrm{C}$. We add a thermocouple to measure the effective temperature with $1^{\circ} \mathrm{C}$ accuracy. The chamber possesses holes above and below the sample, so that the laser beam passes through it.

\subsection{Measurement system}

Through the first coupler $99 / 1,1 \%$ of the emitted power is sent towards a wavelength meter CLUSTER LM007. It measures the wavelength $\lambda$ provided by the tunable laser with an extremely high accuracy, i.e. $0.05 \mathrm{pm}$. The frequency rate of the wavelength measurements is $100 \mathrm{~Hz}$, and its high sensitivity $(0.1 \mu \mathrm{W})$ allow us to use the major part of the power to illuminate the sample. 
To measure the transmittance of the interferometer, we use 4 identical plastic optical fibers with $2 \mathrm{~mm}$ diameter at four defined places, i.e. one at the centre and the 3 others equally disposed around this one, that is on a $11 \mathrm{~mm}$ diameter circle, forming an equilateral triangle. Theses fibers are plugged to 4 identical photodiodes to evaluate the transmittance on each channel, at the wavelength $\lambda$ and temperature $T$.

\subsection{Control system and data operation}

The tunable laser, the wavelength meter, the temperature stabilized chamber are plugged to a PC that controls them and collects the data. A measurement without sample is done at the beginning, in the aim to evaluate after the exact transmittance of the sample for each channel. In addition, we check at the end that the response of the system without the sample is the same as at the beginning.

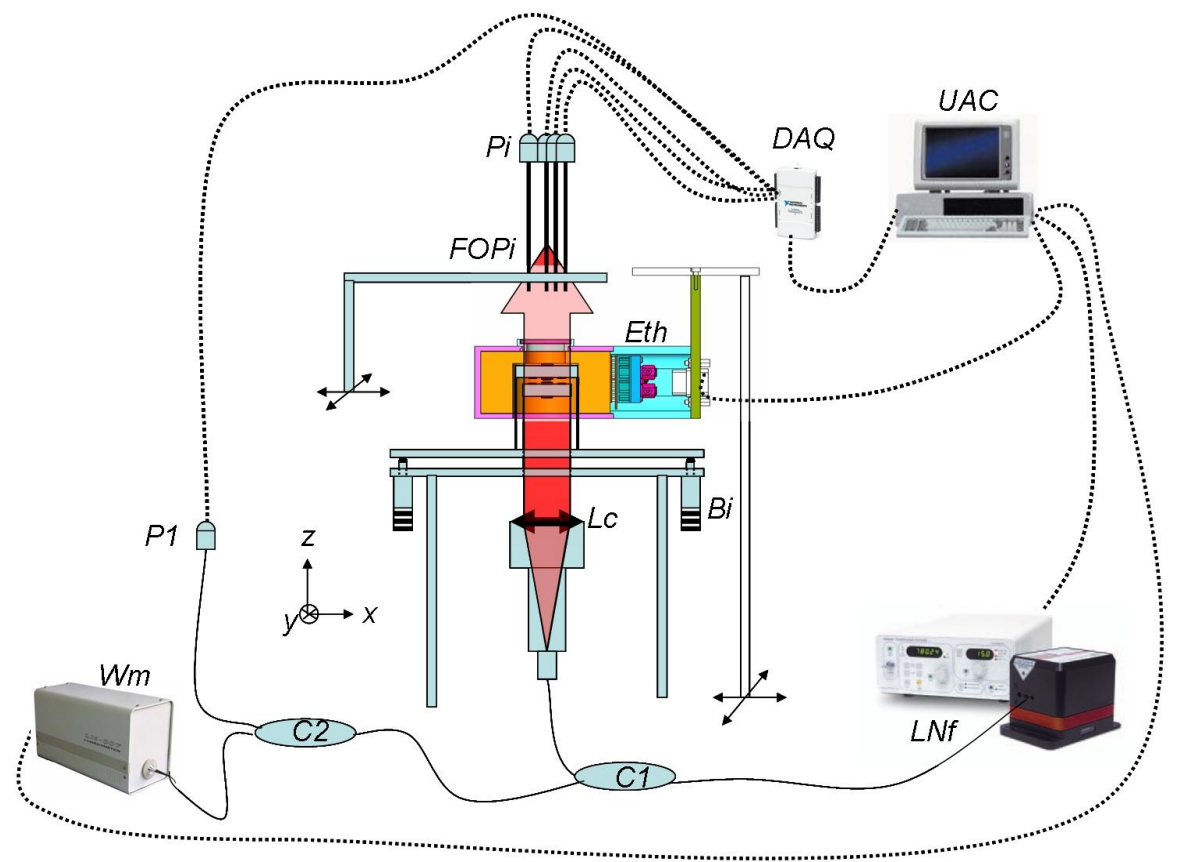

Figure 6: Dedicated set-up for ultrafine measurements of Fabry-Perot resonances

\author{
LNf : Tunable Laser Newfocus TLB6316 \\ Wm : High precision wavelength meter \\ CLUSTER LM007 \\ C1: optical coupler $99 / 1$ \\ C2 : optical coupler $50 / 50$
}

$$
\operatorname{Pi}(i=1 \ldots 5): \text { photodetectors }
$$

Eth : temperature stabilized chamber

FOPi : 4 plastic optical fiber $\varnothing 2 \mathrm{~mm}$

DAQ : data acquisition NI
Lc : collimation lens

Bi : 3 adjusters

Continuous lines: singlemode optical fiber

Dotted underlines : connection cables

\section{PERFORMANCES OF THE SET-UP}

\subsection{Set-up noise}

Figure 7 represents the noise of the system as a function of $\lambda$ without sample, and for a fixed temperature. We evaluate for a channel, the quantity $\operatorname{No}(\lambda)=U\left(t_{1}\right) / U\left(t_{2}\right)$, where $U$ designed the response of the photodiode associated to this channel, and $t_{1}$ and $t_{2}$ designed two different times. We carried out this measurement three times as represented in Fig. 7 
by the different markers. The noise performances of our set-up are quite good, since we obtain an average response very close to 1 , with a standard deviation of only $5.10^{-4}$.

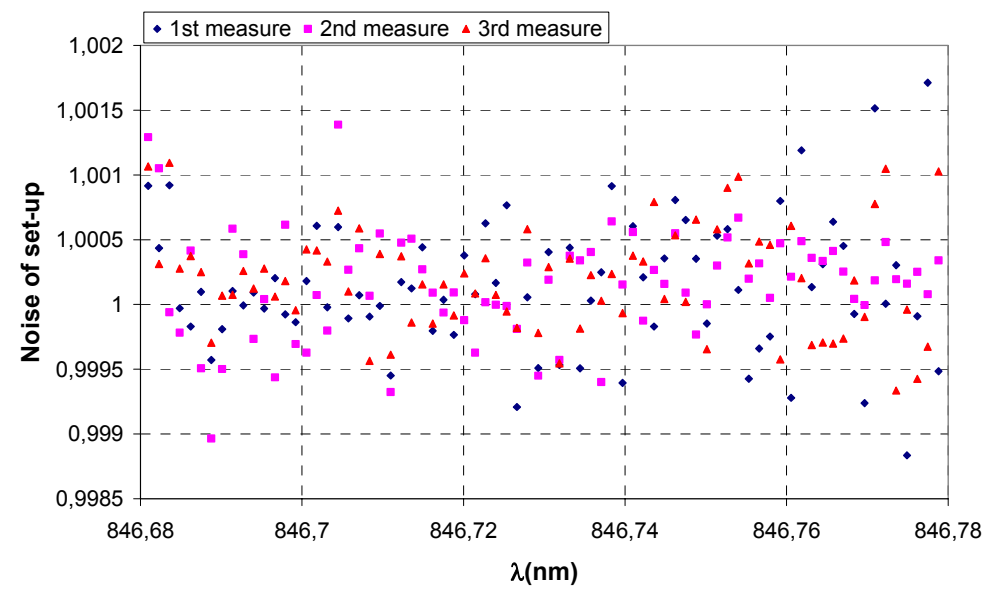

Figure 7: Measured noise on one channel. The different markers corresponds to 3 different measures carried out.

\subsection{Measurements on a BK7 window}

To determine thermal properties of a BK7 window, we chose to follow a maximum of the transmittance $T(\lambda)$. Figure 8 shows the measurement of this function $T(\lambda)$ for the BK7 window at 4 different temperatures. The applied temperature change is at each time only $1^{\circ} \mathrm{C}$, and we measure transmittance peaks shifts of around $7 \mathrm{pm} /{ }^{\circ} \mathrm{C}$. We observe these shifts for a temperature range of around $10^{\circ} \mathrm{C}$, and we evaluate the quantity $\frac{\Delta \lambda}{\lambda}=f(\Delta \theta)$. For this kind of solid-spacer etalon, the shift is induced by refractive index $n$ and thickness $d$ variations, defined by:

$$
\frac{\partial n}{\partial \theta}=\beta n, \text { and } \frac{\partial d}{\partial \theta}=\alpha d
$$

If we consider that the coefficients $\beta$ and $\alpha$ are constant in the temperature range, we get (based on Eq 4 and according to the expressions of $\beta$ and $\alpha$ ):

$$
\frac{\Delta \lambda}{\lambda}=\frac{\Delta n}{n}+\frac{\Delta d}{d}=(\alpha+\beta) \Delta \theta
$$

Figure 9 depicts the calculated function $\frac{\Delta \lambda}{\lambda}=f(\Delta \theta)$. For each point presented in the graph, errors bars are included, but even if we consider $3 \sigma$ errors (and not the standard deviation $\sigma$ ) over 50 measures, these bars are not really visible (the estimated errors are indeed as low as $\pm 1 \cdot 5 \cdot 10^{-7} /{ }^{\circ} \mathrm{C}$ ). Moreover, the slope of this line gives a direct access to the global quantity $(\alpha+\beta)_{\mathrm{BK} 7}$. We evaluated this slope for the 4 channels of our set-up, and we obtained a mean value of 8.7.1 $10^{-6} /{ }^{\circ} \mathrm{C}$, with a standard deviation of $4.10^{-8} /{ }^{\circ} \mathrm{C}$. This average value is in good agreement with $\mathrm{BK} 7$ manufacturer data, which gives a value of $91.10^{-7} /{ }^{\circ} \mathrm{C}$ for the temperature range $\left[-20^{\circ} \mathrm{C}, 70^{\circ} \mathrm{C}\right]$. We realized here a functional qualification for our set-up, i.e. a demonstration that it can be used to precisely evaluate the thermal properties of materials.

At the end, we evaluate the ultimate performance of our bench for the determination of resonances of a low finesse Fabry-Perot interferometer. We obtained a standard deviation of $\mathbf{0 . 1 3} \mathbf{p m}$ on the determination of these maxima for the 50 measures we have done at each temperature, and this result meets the required $0.2 \mathrm{pm}$ specification defined in Section 2 . 


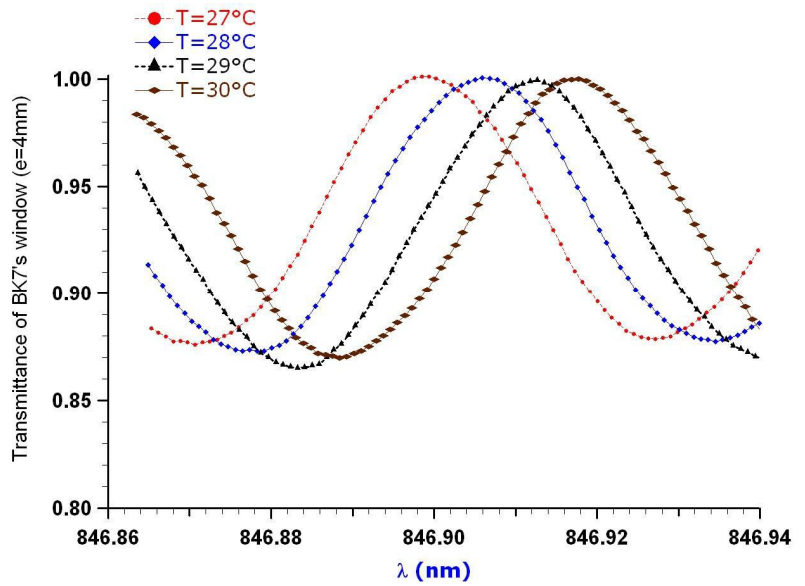

Figure 8: Evolution of the transmittance $T(\lambda)$ for a BK7 window, at different temperatures.

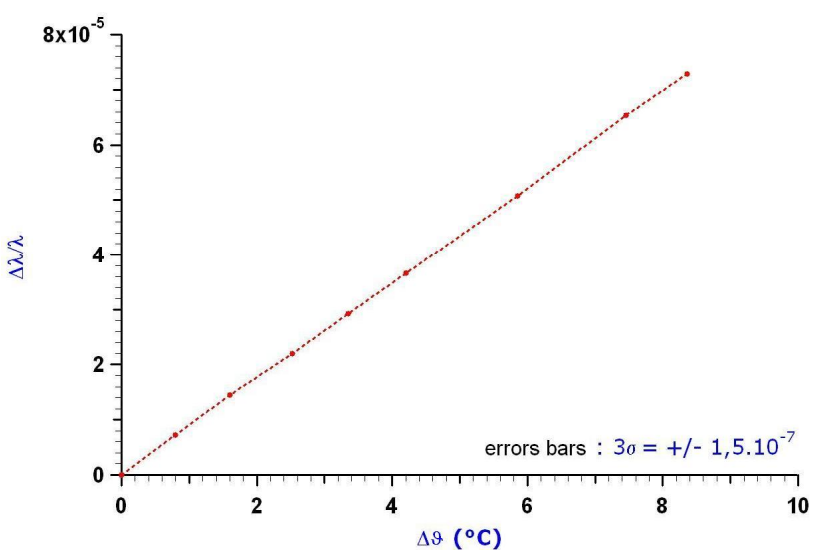

Figure 9: Function $\Delta \lambda / \lambda=\mathrm{f}(\Delta \theta)$ obtained for the BK7 window, on a temperature range around $10^{\circ} \mathrm{C}$. The slope gives the value for the thermal properties of glass.

\subsection{Measurements on an optically contacted ULE Fabry-Perot}

As described in Section 3, we envisage measuring the thermal evolution of an optically contacted ULE Fabry-Perot coated with silver layers. The glass ULE possesses a very small CTE $\left(\alpha_{\mathrm{s}}=0 \pm 3.10^{-8} /{ }^{\circ} \mathrm{C}\right)$ and is therefore the material chosen for this Fabry-Perot. Optical contacting is employed to fixe three ULE plots (thickness $86 \mu \mathrm{m}$, diameter $5 \mathrm{~mm}$ ) to the first ULE window consisting one side of the interferometer (named window A, see Fig. 10). The flatness of the used blocks and windows is better than $\lambda / 10$ PTV. Thereafter, we realize the deposition of silver layers on windows A and B (without blocks). This latter is next attached to window A via the intermediate blocks, using again optical contacting technique. We finally obtain an optically contacted ULE Fabry-Perot with a $86 \mu \mathrm{m}$ thick cavity.

For the silver coating, we decided to deposit only layers where we have our measurements channels in order to limit the deformations induced by the metal. The deposited silver thickness is taken at $40 \mathrm{~nm}$ to assure a good reflectance of both mirrors and to obtain sharp peaks and therefore an enhancement of the sensitivity in our measurements. The different step of manufacturing are schematized in Figure 10, and the optical contacting process, carried out by the company SESO (France), is described in [7].

We can now apply a deterministic thermal change on this Fabry-Perot. As we mentioned before, we are sensitive to the CTE of the material composing the blocks of the Fabry-Perot, i.e. the ULE glass. By comparing the centre with the outer channels, we access to information on the deformation of the structure, due to the CTE difference between the silver coatings and the ULE substrate [8-10].
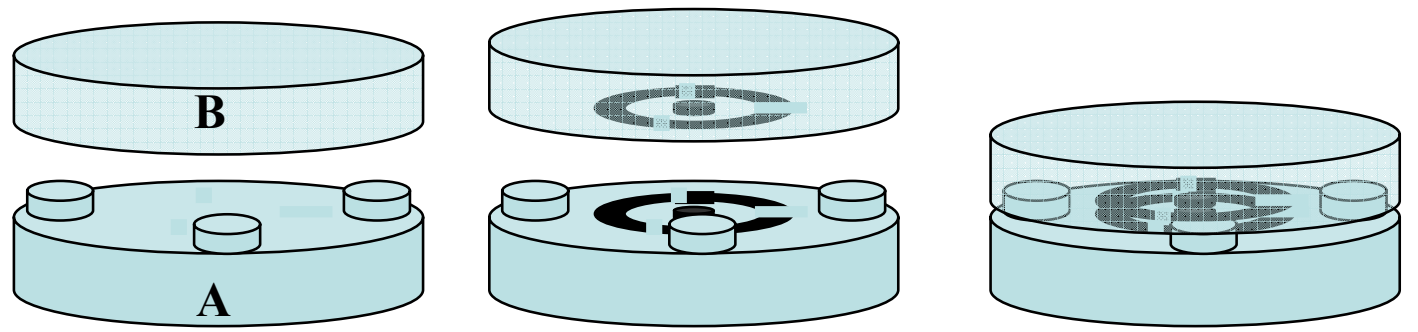

Figure 10: Scheme of the different steps of fabrication to realize an optically ULE Fabry-Perot. First, three blocks (of $5 \mathrm{~mm}$ of diameter and $86 \mu \mathrm{m}$ thick) are fixed by optical contacting on window A. Second, we deposit partial silver coatings (to minimize deformation when we apply a thermal change on the structure). At last, window B is fixed to the three blocks to form an optically contacted Fabry-Perot. 
Figure 11 shows the comparison between the relative wavelengths shifts measured at the center of the contacted FabryPerot and the previously studied BK7 window. We observe the effect of the low CTE of ULE glass, since the CTE of ULE is 25 times less than those of BK7. Our set-up allows also to evaluate the deformation of the structure due to the CTE difference between silver coatings and the substrate. Figure 12 compares the behavior of outer channels with the center. For each channel noted $i$, we have:

$$
\left(\frac{\Delta \lambda}{\lambda}\right)_{i}=\left(\frac{\Delta n_{F P}}{n_{F P}}+\frac{\Delta D_{F P}}{D_{F P}}\right)_{i}
$$

Figure 12 shows the evolution versus the temperature change $\Delta \mathrm{T}$ of the following quantity:

$$
\overline{\left(\frac{\Delta \lambda}{\lambda}\right)_{\text {periph }}}-\left(\frac{\Delta \lambda}{\lambda}\right)_{\text {center }}=\left(\frac{\overline{\Delta D_{F P}}}{D_{F P}}\right)_{\text {peripheral }}-\left(\frac{\Delta D_{F P}}{D_{F P}}\right)_{\text {center }}
$$

where the symbol with bar represents the mean value.

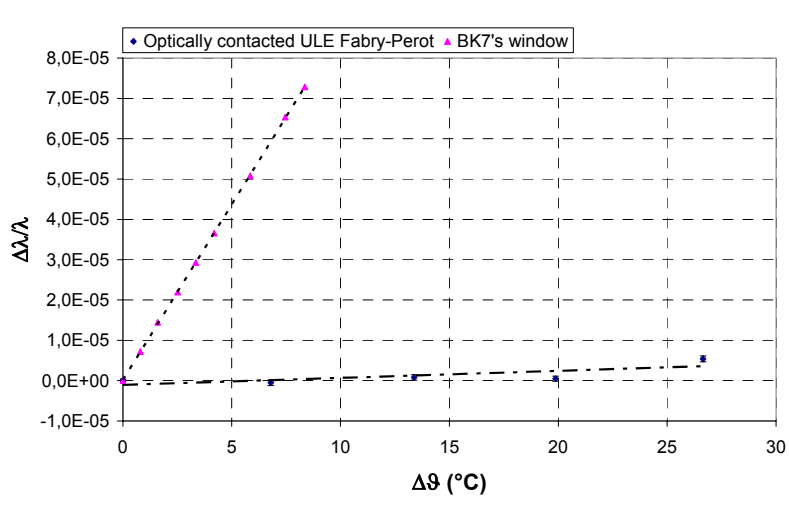

Figure 11: Comparison of the relative shift at the center of the structure between the optically contacted Fabry-Perot and a BK7 window

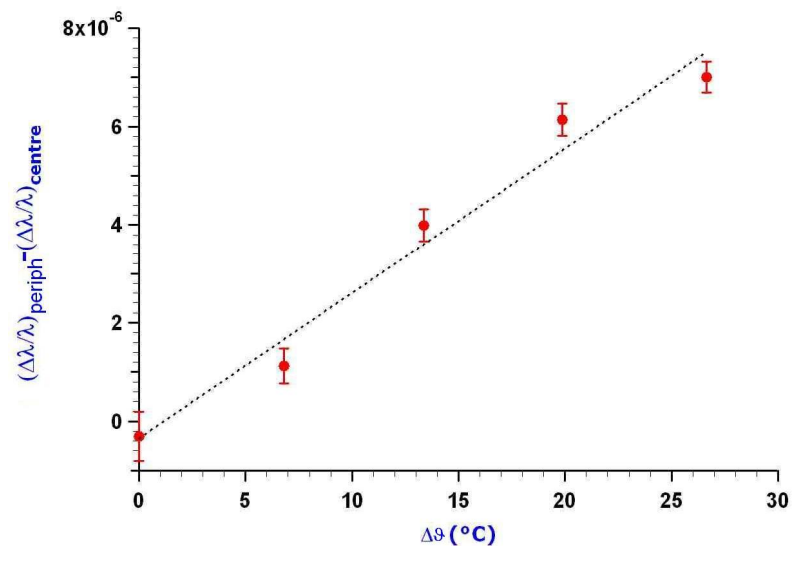

Figure 12: Experimentally measured deformations between the outer channels and the center

We compare the results given by Fig. 12 with a simulation by Finite Element Method taking account the deformation of the interferometer. This simulation allows us to conclude that the interferometer undergoes deformations a lot more than we expected (the slope of the difference between the peripheral and center channels are 20 times more than simulations predict). This result brings us to investigate with more attention the behavior of deformation due to CTE difference between materials, see reference [5].

\section{CONCLUSION AND PERSPECTIVES}

The knowledge of thin film deformations is of a great interest nowadays, although difficult to dissociate from the usual substrate support the coatings. We investigated a dedicated set-up to measure the resonances of a Fabry-Perot interferometer in several places with an accuracy of at least $0.2 \mathrm{pm}$. These measurements are used to follow the wavelengths shifts observed by the interferometer under a temperature change. Under this thermal change, the wavelengths shifts are directly connected to the optical thickness variation of the interferometer cavity. This ultrafine measurement was used to determine thermal properties of materials used to compose the cavity.

In addition, we employed our bench to determine the deformation of an optically contacted ULE Fabry-Perot introduced by the deposit of silver coatings. We concluded that the deformation is to be more important than we expected by simulations of the thermal behavior using the Finite Element Method. This question is still under investigation. 
Finally, to improve the knowledge on deformations of thin films deposited on substrate, we envisage an evolution of our set-up by adding a Charge-Coupled Detector to replace our optical fibers-photodiodes assemblies. With such a system of data collection, we should then access to a global view of the deformation of surfaces.

\section{REFERENCES}

[1] A. White., "Use of Cer-Vit Material in Low Expansion Reference Optical Cavities, ” Appl. Optics, 6, 1138 (1967)

[2] S.F. Jacobs, J.N. Bradford, and J.W. Berthold, "Ultraprecise Measurement of Thermal Coefficients of Expansion." Appl. Opt., 9(11), 2477-2480 (1970).

[3] H.A. Macleod, "Thin Film optical Filters," 3rd ed. 260-264 (1986).

[4] M. Born, and E. Wolf, "Principle of optics," 6th ed. 329-333 (1980)

[5] S. Michel, "Determination of thermal and elastic coefficients of optical thin-film materials," Proceedings of SPIE, (7101) (2008).

[6] J.A. Stone, and J.H. Zimmerman. "Index of Refraction of Air," calculation and details available on: http://emtoolbox.nist.gov/Wavelength/Documentation.asp. (2002).

[7] J.J. Fermé, Optical Contacting, http://www.seso.com/pub/optical\%20contacting.pdf

[8] A. Ennos, "Stresses developed in Optical Film Coating," Applied Optics 5(1), 51-62.(1966)

[9] C.H. Hsueh, "Thermal Stresses in elastic multilayer systems," Thin Solid Films. 418, 182-188 (2002)

[10] S. Tamulevicius, "Stress and strain in the vacuum deposited thin films," Vacuum. 51(2), 127-139 (1998) 